\title{
R10 - APLICAÇÃO DA CITOMETRIA DE FLUXO NA AVALIAÇÃo DE CONJUGADOS FLUORESCENTES UTILIZADOS EM IMUNOENSAIOS
}

$\underline{\text { Marta de Almeida Santiago }}{ }^{1}$, Bruna de Paula Fonseca e Fonseca ${ }^{1}$, Christiane de Fátima da Silva Marques ${ }^{1}$, Edimilson Domingos da Silva ${ }^{1}$, Álvaro Luiz Bertho ${ }^{2}$, Ana Cristina Martins de Almeida Nogueira ${ }^{3}$.

1. Laboratório de Tecnologia Diagnóstica, Vice-Diretoria de Desenvolvimento Tencnológico, Bio-Manguinhos, FIOCRUZ.

2. Laboratório de Imunoparasitologia \& Plataforma de Citometria de Fluxo, Instituto Oswaldo Cruz, FIOCRUZ.

3. Laboratório de Biotecnologia e Fisiologia de Infecções Virais, Instituto Oswaldo Cruz, FIOCRUZ.

Objetivos: O presente trabalho teve como objetivo avaliar a aplicação da citometria de fluxo como ferramenta para monitoramento de conjugados utilizados em imunoensaios e como parâmetro de acompanhamento da estabilidade desses insumos. Tais resultados fazem parte do projeto de tese de doutorado de Marta de Almeida Santiago intitulado "Aplicação da citometria de fluxo no controle de qualidade de insumos e processos utilizados no desenvolvimento de kits para diagnóstico", do curso de Pós-graduação em Vigilância Sanitária do INCQS.

Metodologia: Microesferas magnéticas (xMAP®, Luminex, Austin) foram acopladas com diferentes concentrações de anti-IgG humana $(5,2,5,1$ e 0,5ug/ mL) e anti-HBS $(1: 300,1: 1000$ e 1:3000) conjugadas à ficoeritrina $(\mathrm{PE})$ e submetidas a análise citofluorimétrica no citômetro de fluxo BD FACSCalibur (Becton \& Dickinson) do Laboratório de Tecnologia Diagnóstica (LATED), Bio-Manguinhos. O protocolo de aquisição foi realizado utilizando o programa CellQuest (BD) e as análises dos resultados foram feitas através do software FlowJo (Tree Star Inc, USA). Para cada amostra foram adquiridos 2000 eventos na região ("gate") de microesferas. Os conjugados foram avaliados quanto ao seu perfil de marcação, média geométrica e coeficiente de variação (CV) em histogramas de leitura no canal de fluorescência com filtro 585/42 (FL2 / PE).

Resultados: Não houve diferença no grau de acoplamento quando foram utilizados diferentes códigos de microesferas (\#12, \#35 e \#70). A avaliação das concentrações dos 
conjugados demonstrou que no caso dos conjugados S1, S4 e M2 a melhor concentração de acoplamento foi 5ug/ mL, enquanto para F2 e C1 a diluição 1:300 da solução estoque apresentou melhores resultados. Em tais concentrações, os conjugados apresentaram as maiores médias geométricas (maior grau de marcação) e os menores coeficientes de variação (maior homogeneidade de acoplamento). Em relação ao acompanhamento da estabilidade dos conjugados, observou-se uma tendência à redução das médias geométricas nos conjugados S2 e S3 ao longo do tempo de avaliação, tendência esta não observada nos outros conjugados estudados.

Conclusão: $\mathrm{O}$ acoplamento dos conjugados às microesferas demonstrou ser uma boa ferramenta para avaliação direta desses insumos pela citometria de fluxo.

Os resultados observados sugerem que conjugados do mesmo fabricante podem apresentar variações significativas de estabilidade lote a lote, demonstrando a importância deste acompanhamento durante o processo produtivo de kits para imunoensaios.

O acompanhamento da estabilidade de insumos pela citometria de fluxo poderá contribuir de forma significativa para o estabelecimento de metodologias de controle de qualidade de insumos e processos utilizados em imunoensaios. 